\title{
Increase in coleoptile length and establishment by LCOl-A1, a genetic locus with major effect in wheat
}

\author{
William D. Bovill ${ }^{1 *}$ (D, Jessica Hyles ${ }^{1}$, Alexander B. Zwart², Brett A. Ford ${ }^{1}$, Geetha Perera', Tanya Phongkham', \\ Brenton J. Brooks', Gregory J. Rebetzke ${ }^{1}$, Matthew J. Hayden³, James R. Hunt ${ }^{4}$ and Wolfgang Spielmeyer ${ }^{1 *}$
}

\begin{abstract}
Background: Good establishment is important for rapid leaf area development in wheat crops. Poor establishment results in fewer, later-emerging plants, reduced leaf area and tiller number. In addition, poorly established crops suffer from increased soil moisture loss through evaporation and greater competition from weeds while fewer spikes are produced which can reduce grain yield. By protecting the emerging first leaf, the coleoptile is critical for achieving good establishment, and its length and interaction with soil physical properties determine the ability of a cultivar to emerge from depth.
\end{abstract}

Results: Here we characterise a locus on chromosome 1AS, that increases coleoptile length in wheat, which we designate as LCOI-A1. We identified LCOI-A1 by bulked-segregant analysis and used a Halberd-derived population to fine map the gene to a $2 \mathrm{cM}$ region, equivalent to $7 \mathrm{Mb}$ on the IWGSC genome reference sequence of Chinese Spring (RefSeqv1.0). By sowing recently released cultivars and near-isogenic lines in the field at both conventional and deep sowing depths, we confirmed that Locl-A1 was associated with increased emergence from depth in the presence and absence of conventional dwarfing genes. Flanking markers IWB58229 and IWA710 were developed to assist breeders to select for long coleoptile wheats.

Conclusions: Increased coleoptile length is sought in many global wheat production areas to improve crop emergence. The identification of the gene Lcol-A1, together with tools to allow wheat breeders to track the gene, will enable improvements to be made for this important trait.

Keywords: Triticum aestivum, Wheat, Coleoptile, Emergence, Molecular marker, SNP

\section{Background}

In water-limited environments with high evaporative demand, wheat seedlings need to emerge and develop leaf area rapidly to allow good establishment. Poor establishment reduces the number of spikes per square meter and grain yield [32, 37]. By protecting the emerging first leaf, the coleoptile is critical for achieving good establishment, and its length and interaction with soil physical properties determine the ability of a cultivar to emerge from depth $[11,12,30]$.

Longer coleoptiles are beneficial in many agricultural systems worldwide, particularly where deep sowing into

\footnotetext{
* Correspondence: William.Bovill@csiro.au; Wolfgang.Spielmeyer@csiro.au ${ }^{1}$ CSIRO Agriculture and Food, P.O. Box 1700, Canberra ACT 2601, Australia Full list of author information is available at the end of the article
}

moisture is required [20,38,44], where stubble retention practices have been adopted [34] or where crops are sown early into warmer soils $[7,36]$. In Australia, there exists a narrow period during which crops must flower in order for yields to be maximised [18], and crop yield is thus very sensitive to timing of establishment. There is an emerging trend to sow crops earlier into warmer soils to optimise whole-farm logistics and increase water-use efficiency $[16,17,19,28]$, but seed-bed soil water potentials are often sub-optimal and temperatures supraoptimal for germination and emergence at this time. The lack of surface moisture during the short, optimum sowing window encourages farmers to sow deep, but if the coleoptile is short, the first leaf may not emerge or may be damaged, leading to poor establishment $[38,44]$.

(c) The Author(s). 2019 Open Access This article is distributed under the terms of the Creative Commons Attribution 4.0 International License (http://creativecommons.org/licenses/by/4.0/), which permits unrestricted use, distribution, and 
Many current cultivars are not be suited to deep sowing due to their short coleoptiles, and warm soils exacerbate this by further shortening coleoptile length [36]. Long coleoptile wheats would provide growers with more flexibility to sow deep, but at present a lack of knowledge about genes that promote coleoptile growth and efficient selection tools prevent breeders from incorporating this important trait.

Most semi-dwarf cultivars grown worldwide have relatively short coleoptiles due to the presence of dwarfing genes $R h t-B 1 b$ and $R h t-D 1 b$ [23]. These genes encode mutant DELLA proteins that are negative regulators of growth with a large negative effect on coleoptile length which is associated with poor establishment in the field $[1,2,15,42]$. Despite this, several studies have found variation for coleoptile length within semi-dwarf wheats, suggesting that loci which increase coleoptile length can be selected for in semi-dwarf backgrounds $[1,5]$.

The search for genetic variation of increased coleoptile length has progressed from phenotypic screening to genome-wide approaches including QTL mapping and genome-wide association studies (GWAS). Preliminary QTL studies focussed on interval mapping approaches in bi-parental populations with sparse maps constructed using AFLP or microsatellite markers [27, 31, 33, 40, 41, 45]. This approach identified multiple QTL with generally small effect (explaining $<5 \%$ of phenotypic variation) and large confidence intervals. The advent of cost-effective high density SNP markers and more complex population structures has enabled greater mapping resolution. Rebetzke et al. [35], using a 4-way multi-parent advanced generation intercross (MAGIC) wheat population genotyped with the $9 \mathrm{~K}$ SNP array [10], identified seven QTL, while GWAS on a set of 893 wheat accessions also genotyped with the $9 \mathrm{~K}$ SNP array identified eight QTL for coleoptile length [26]. Both mapping studies identified QTL on either or both of chromosomes $4 \mathrm{~B}$ and $4 \mathrm{D}$ associated with the dwarfing genes $R h t-B 1 b$ and $R h t-D 1 b$, confirming the negative effect of these genes on coleoptile length, but also identified loci independent of the dwarfing genes which increased coleoptile length.

Previous studies highlight that coleoptile length is controlled by many genes with relatively small effects. This study reports the identification, fine mapping, and field validation of a coleoptile length-promoting gene on chromosome $1 \mathrm{~A}$ of bread wheat. We show that the gene promotes coleoptile length in current elite germplasm, and that longer coleoptiles result in better emergence in both tall and semi-dwarf wheat lines when sown deep in the field.

\section{Results}

Genomic regions associated with long coleoptiles Halberd and Uruguay386 are tall wheats known for their long coleoptiles. To identify genomic regions contributing to the trait, the coleoptile length was measured in $\mathrm{BC}_{1} \mathrm{~F}_{2}$ lines from two backcross-derived populations of Halberd or Uruguay386 to the short coleoptile Chinese wheat line CM18. As expected, Halberd and Uruguay displayed a long coleoptile phenotype and CM18 a short coleoptile phenotype (Fig. 1). Lines with extremely long and short coleoptiles were selected from these populations for bulked-segregant analysis (BSA) using the $9 \mathrm{~K}$ wheat SNP array [10]. Marker-trait associations (MTAs) were detected on 13 wheat chromosomes in the Halberd*2/CM18
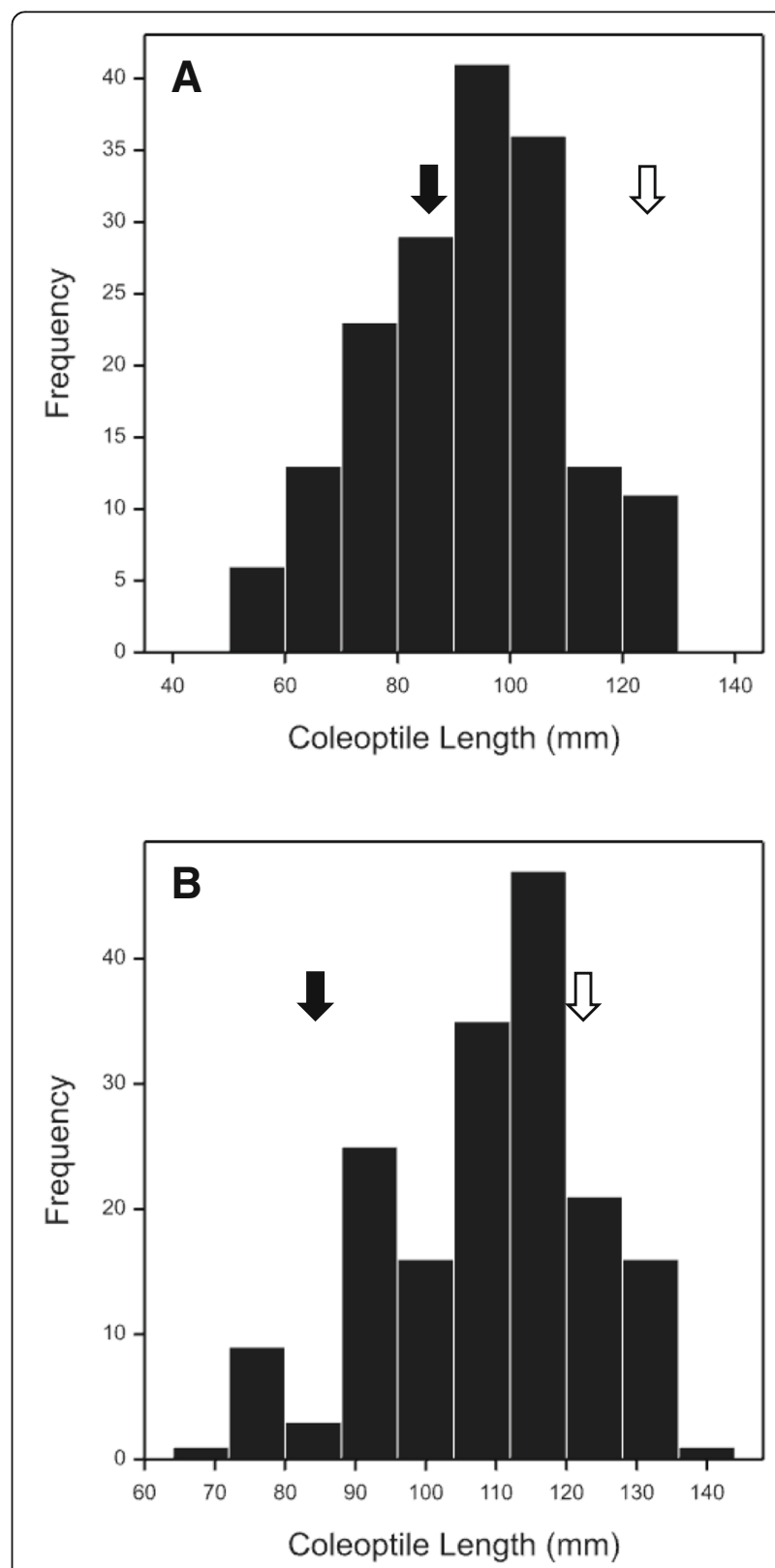

Fig. 1 Phenotypic distribution of coleoptile length in $188 B_{1} F_{2}$ lines from each of the Halberd*2/CM18 population (a) and the Uruguay $386^{*} 2 / \mathrm{CM} 18$ populations (b). Parental means are indicated by filled (CM18) and unfilled (Halberd and Uruguay) arrows 
population, and 8 chromosomes in the Uruguay*2/CM18 population (Additional file 1: Table S1). Because a large number of tightly linked SNP markers on chromosome 1A were strongly associated with the trait in both populations (Table 1), it was decided to genetically map the trait in this region.

\section{Fine-mapping of the $1 \mathrm{~A}$ long coleoptile region}

For genetic mapping of the trait on chromosome 1A, a new backcross-derived population was developed from HI25M (a Halberd derivative) and Young that lack the $R h t-B 1 b$ dwarfing gene. Fifty-four $\mathrm{BC}_{1} \mathrm{~F}_{2}$ plants were genotyped with IWA164, a $1 \mathrm{~A}$ marker selected from the $1 \mathrm{~A}$ region in the $9 \mathrm{~K}$ SNP consensus map [10]. The association between marker IWA164 and the trait was confirmed after $\mathrm{BC}_{1} \mathrm{~F}_{3}$ families were phenotyped for coleoptile length, with the marker explaining approximately $30 \mathrm{~mm}$ of phenotypic variation for coleoptile

Table 1 Significant $(p<0.0001)$ SNPs on chromosome $1 \mathrm{~A}$ detected from the bulked-segregant analysis (BSA) in the Halberd*2/CM18 and Uruguay386*2/CM18 populations. Locations (_Loc; CM) from the $9 \mathrm{~K}$ [10] and $90 \mathrm{~K}$ [43] consensus maps are provided. NA = SNP not placed on consensus map

\begin{tabular}{|c|c|c|c|}
\hline SNP Name & SNP ID & 9K_LOC & 90K_LoC \\
\hline \multicolumn{4}{|l|}{ Halberd*2/CM18 } \\
\hline wsnp_Ku_c17726_26872129 & IWA6636 & 51.12 & 71.05 \\
\hline wsnp_Ex_c3253_5995011 & IWA3399 & 54.28 & NA \\
\hline wsnp_Ex_c6826_11774795 & IWA4578 & 57.95 & 70.10 \\
\hline wsnp_Ku_c3468_6420199 & IWA6942 & 57.95 & 70.10 \\
\hline wsnp_Ex_c3747_6824863 & IWA3612 & 57.95 & 70.10 \\
\hline wsnp_Ex_c8364_14095508 & IWA4797 & 62.39 & 71.10 \\
\hline wsnp_Ku_c37925_46679146 & IWA6972 & 70.70 & 71.10 \\
\hline wsnp_Ku_c33917_43336069 & IWA6934 & 109.32 & 101.19 \\
\hline \multicolumn{4}{|l|}{ Uruguay386*2/CM18 } \\
\hline wsnp_Ex_c5323_9408829 & IWA4163 & 35.82 & 51.09 \\
\hline wsnp_Ku_c11896_19337444 & IWA6441 & 39.00 & NA \\
\hline wsnp_BE586140A_Ta_2_1 & IWA360 & 40.65 & 55.18 \\
\hline wsnp_Ex_c34821_43076533 & IWA3499 & 51.12 & 71.48 \\
\hline wsnp_Ex_c3253_5995011 & IWA3339 & 54.28 & NA \\
\hline wsnp_Ex_c2749_5091813 & IWA3115 & 56.97 & 70.10 \\
\hline wsnp_JD_rep_c49359_33578909 & IWA6260.1 & 57.95 & 70.10 \\
\hline wsnp_Ex_rep_c104050_88861052 & IWA5109 & 57.95 & 70.10 \\
\hline wsnp_Ra_c26956_36503468 & IWA7804 & 57.95 & 70.10 \\
\hline wsnp_Ex_c2389_4477096 & IWA2847 & 57.95 & 70.10 \\
\hline wsnp_Ex_c3142_5808330 & IWA3338 & 64.39 & 70.10 \\
\hline wsnp_BE518393A_Td_2_3 & IWA352 & 64.39 & 70.10 \\
\hline wsnp_Ex_rep_c66382_64577768 & IWA5226 & 67.01 & 70.10 \\
\hline wsnp_CAP11_c1029_611774 & IWA639 & 68.32 & 70.10 \\
\hline wsnp_Ku_rep_c71909_71634013 & IWA7505 & 68.65 & 70.79 \\
\hline
\end{tabular}

length in this population (Fig. 2). Additional SNP-based markers IWA3374 and IWA5435 were selected from the $1 \mathrm{~A}$ consensus map and shown to flank a genetic interval $(\sim 10 \mathrm{cM})$ which contained a gene that will be referred to as Lcol-A1 (Fig. 3).

To refine the map location of Lcol-A1, flanking markers IWA3374 and IWA5435 were converted to KASP assays and used to screen $900 \mathrm{BC}_{2} \mathrm{~F}_{2}$ equivalents from the Young*3/HI25M population to identify 204 recombinants between IWA3374 and IWA5435. These recombinants were genotyped with IWA164 and a subset of 28 lines were selected for phenotyping. To improve phenotyping accuracy, recombination events were fixed by genotyping $\mathrm{BC}_{2} \mathrm{~F}_{3}$ progeny and selecting segregants that were homozygous at flanking marker loci. Coleoptile length was measured on $\mathrm{F}_{4}$ families derived from homozygous recombinants to map Lcol-A1 to a $4 \mathrm{cM}$ interval flanked by markers IWA164 and IWA5435 (Fig. $3)$. An additional seven markers were added to the target interval by screening parental lines with the $90 \mathrm{~K}$ SNP array [43] and genotyping recombinants with polymorphic markers. In a second round of fine mapping, a further 57 recombinants (from total of 204) were selected based on IWA164 and IWA5435 marker genotypes to measure coleoptile length on $\mathrm{BC}_{2} \mathrm{~F}_{4}$ homozygous, recombinant families. Consequently the map location of Lcol-A1 was refined to the $2 \mathrm{cM}$ marker interval between IWB58229-IWA710 which corresponded to approximately

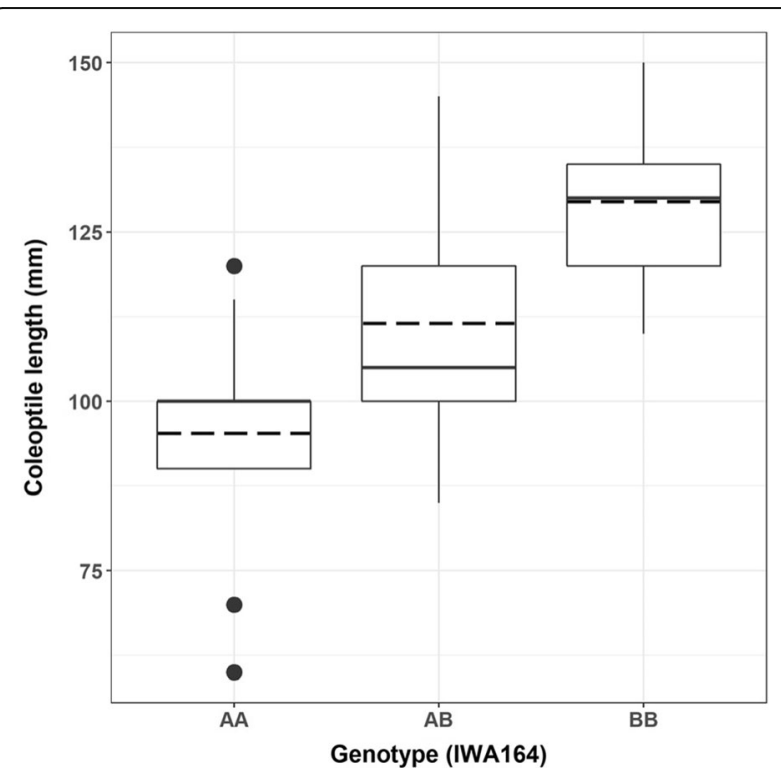

Fig. 2 Association between genotype at marker IWA164 and coleoptile length of $\mathrm{BC}_{1} \mathrm{~F}_{3}$ seedlings from the Young*2/H125M. $\mathrm{AA}$, homozygous negative; $A B$, heterozygous; $B B$ homozygous positive. The lower and upper edges of the box represent 25th and 75th percentiles, interior solid lines denote the median and dashed lines denote the mean. Potential outliers are plotted as black dots, and identified according to the $1.5 \times$ interquartile range criterion 


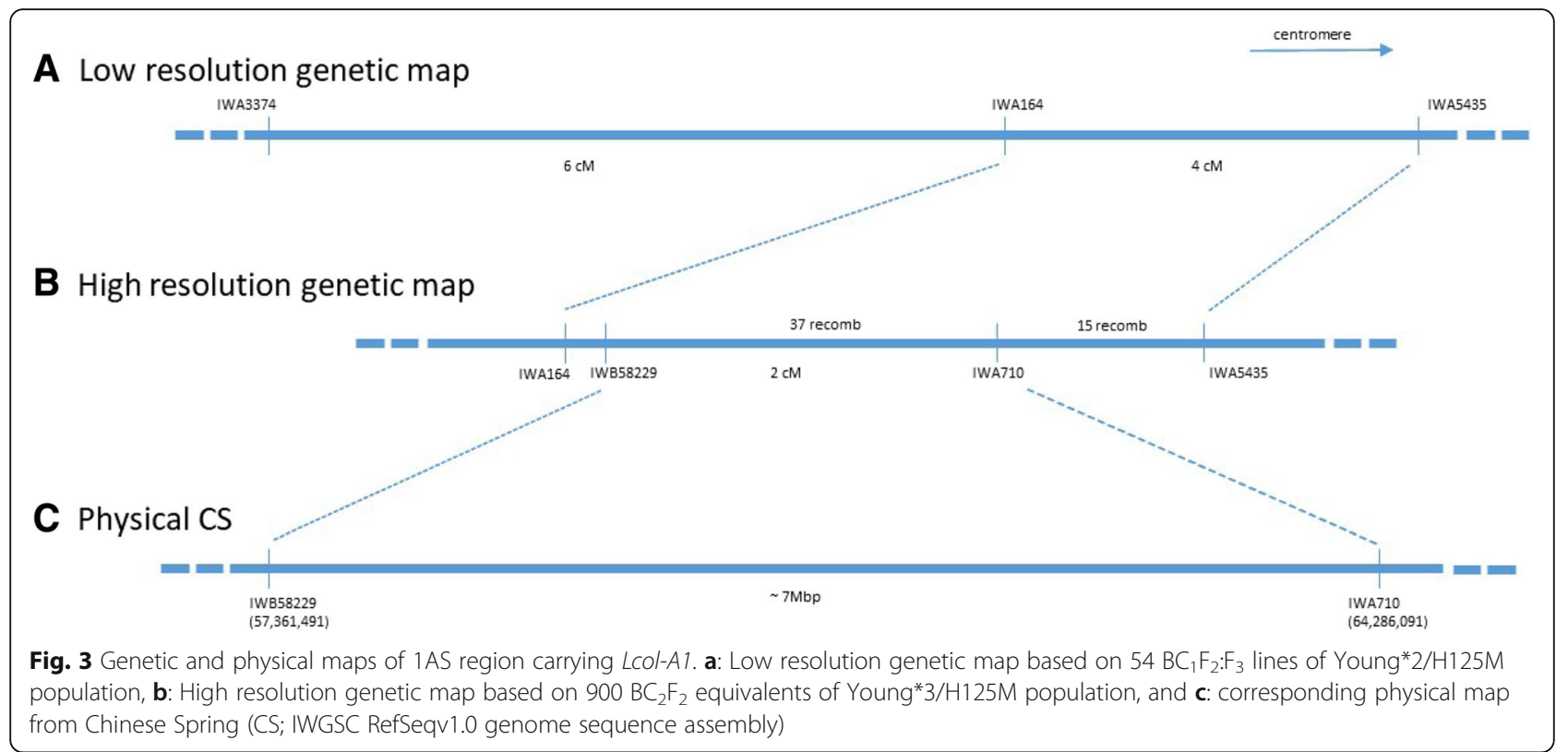

$7 \mathrm{Mb}$ of physical distance in the RefSeqv1.0 genome sequence assembly [3].

\section{Validation of Lcol-A1 in Espada genetic background}

The effect of Lcol-A1 was confirmed by transferring the long coleoptile haplotype from HI25M into the Australian cultivar Espada. In a backcross-derived population (Espada*4/HI25M) which lacked the $R h t$-D1b dwarfing gene from Espada, coleoptile length was continuously distributed ranging from 65 to $140 \mathrm{~mm}$ in length (Fig. 4). The same marker IWA164 which was linked to the trait in the Young background explained approximately 25 $\mathrm{mm}$ of variation in homozygous lines carrying different haploytypes in the Espada background. These results confirm that Lcol-A1 contributes to coleoptile growth in another genetic background indicating a major gene for coleoptile growth has been identified.

\section{Lcol-A1 contributes to better emergence in the field}

To show that the long coleoptile trait conferred by Lcol$1 A$ also improves plant emergence and establishment under field conditions, we tested the hypothesis that cultivars with Lcol-A1 produce longer coleoptiles and emerge better when sown deep under field conditions compared to cultivars that lack the long coleoptile allele of the gene. Five cultivars that were predicted to carry the long allele based on the haplotype of flanking markers and pedigree and six that were predicted to carry the short allele were selected to test the hypothesis (Table 2). These released cultivars represented a wide range of genetic diversity of the Australian genepool. To determine the effect of Lcol-A1 in tall background, isolines from the Young*3/HI25M population (five short and five long haplotype) were also evaluated for emergence under field conditions.

Prior to field testing, we confirmed that Lcol-A1 contributed to coleoptile length in semi-dwarf cultivars which were included in field trials. The coleoptile length, when measured under controlled conditions, was approx. $18 \%$

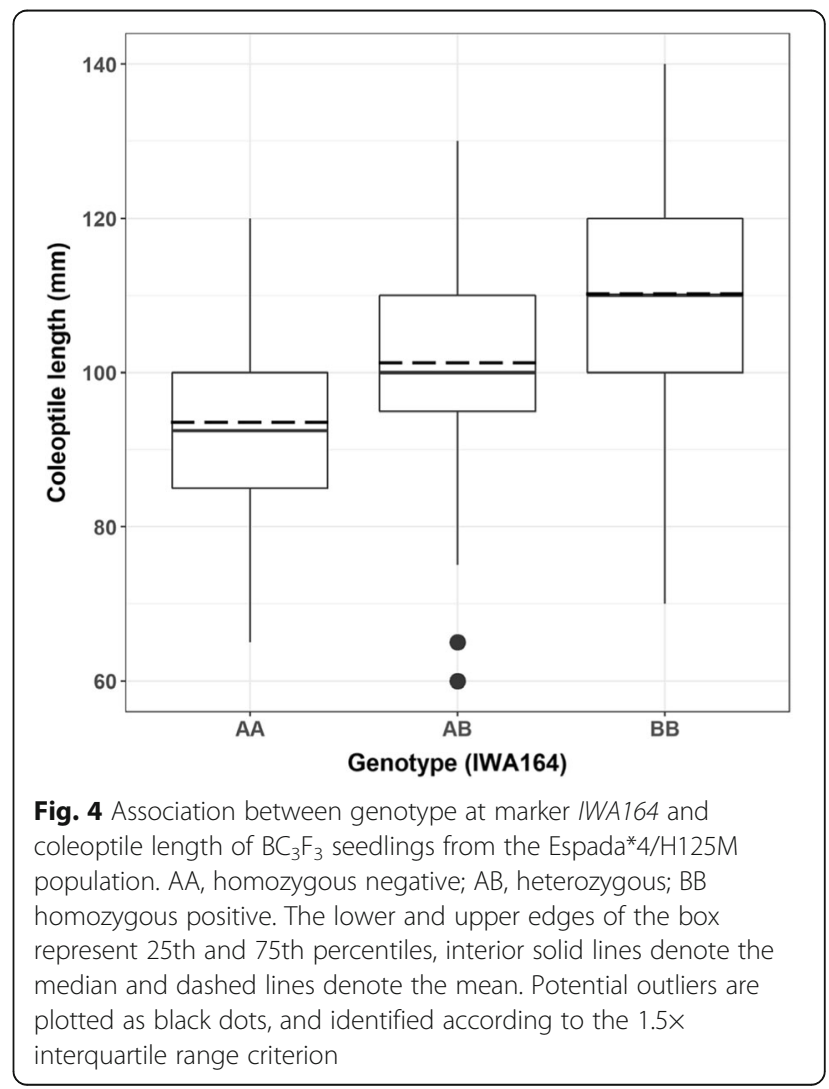


Table 2 Cultivars selected for examining the effect of $L C O /-A 1$ on emergence in the field. Lcol-A1 haplotype and year of release (Year) are indicated. Pedigree information was retrieved from the Genetic Resources Information System for Wheat and Triticale (GRIS; wheatpedigree.net)

\begin{tabular}{llll}
\hline Cultivar & Lcol-A1 Haplotype & Year & Pedigree \\
\hline EGA Gregory & Short & 2004 & Pelsart/3*Batavia \\
Emu Rock & Short & 2011 & Westonia/Kukri/Perenjori/Ajana \\
Espada & Short & 2008 & RAC-875/Krichauff//Excalibur/Kukri/3/RAC-875/Krichauff/4/RAC-875//Excalibur/Kukri \\
Mace & Short & 2008 & Wyalkatchem/Stylet//Wyalkatchem \\
Suntop & Short & 2011 & Sunco/2*Pastor//SUN-436-E \\
Young & Short & 2005 & VPM-1/3*Beulah//Silverstar \\
Excalibur & Long & 1990 & RAC-177/Uniculm-492//RAC-311-S \\
Magenta & Long & 2007 & Carnamah/Tammin-18 \\
Phantom & Long & 2010 & Sentine**3/Yitpi \\
Scout & Long & 2009 & Sunstate/QH-76-1//Yitpi \\
Yitpi & Long & 2000 & C-8-MMC-8-HMM/Frame
\end{tabular}

Significant $(\mathrm{p}<0.0001)$ SNPs on chromosome $1 \mathrm{~A}$ detected from the bulked-segregant analysis (BSA) in the Halberd/CM18 and Uruguay386/CM18 backcross populations. Locations (_Loc; CM) from the $9 \mathrm{~K}$ [10] and $90 \mathrm{~K}$ [43] consensus maps are provided. NA = SNP not placed on consensus map

longer in cultivars predicted to carry the long Lcol-A1 allele (Fig. 5). In tall isolines the final length increased compared to the cultivars but the difference between genotypes also increased to approximately $25 \%$ consistent with an expected growth promotion effect in the absence of conventional dwarfing genes.

For field validation, cultivars were sown at depths of $25 \mathrm{~mm}$ and $70 \mathrm{~mm}$ representing normal and deep sowing treatments at Yanco in western NSW (Australia) in 2016 and 2017. Emergence was counted in regular intervals during the first 5 weeks of the experiments and the mean emergence counts were plotted for both sowing depths and genotype categories (Fig. 6). In both years, more plants emerged from the deep sowing treatment in cultivars with the long Lcol-A1 allele than in cultivars with the short allele. The difference in emergence from deep sowing was more pronounced in the Young*3/ HI25M isolines, confirming the positive effect of the gene under field conditions. Interestingly, the increase in emergence afforded by Lcol-A1 was apparent in cultivars in 2016 and in isolines in both 2016 and 2017 under the normal sowing depth as well.

\section{Prevalence of Lcol-A1 in an Australian diversity panel}

To assess the prevalence of Lcol-A1 in Australian germplasm, a panel of 197 cultivars and lines that were released from the year 1890 to 2015 and represent a wide range of the Australian gene pool, were

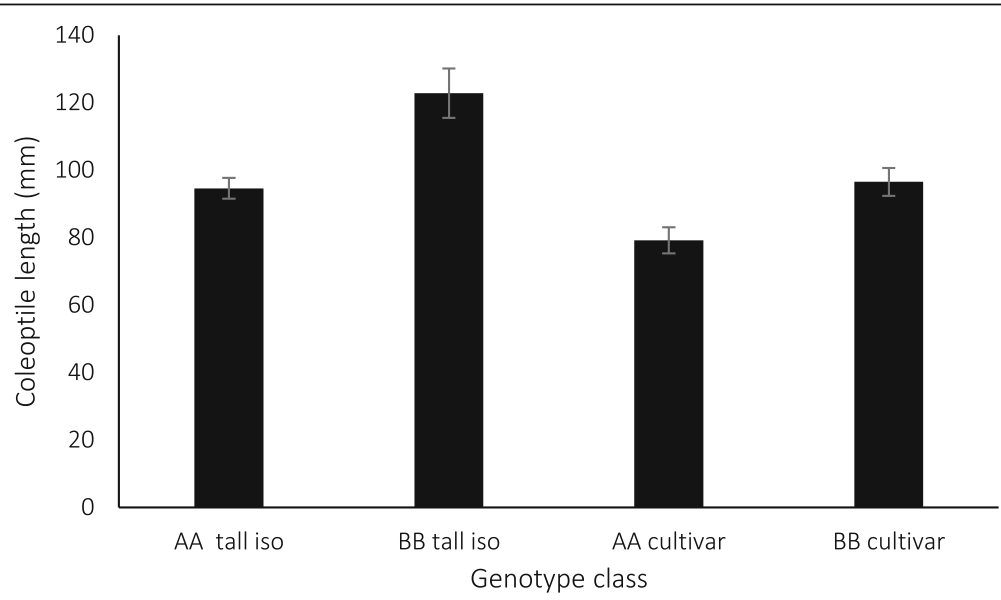

Fig. 5 Coleoptile length of isolines (iso) and cultivars with long (BB) and short (AA) Lcol-A1 alleles under controlled conditions. Data are means $(n=5$ for each isoline class; 6 for the short [AA] cultivar class; and 5 for the long [BB] cultivar class, obtained by averaging values from varieties within the relevant [isoline vs cultivar] genotype class) \pm standard error; differences within genotype class are significant $(p=0.011$ for cultivars, $p<0.01$ for tall isolines) 


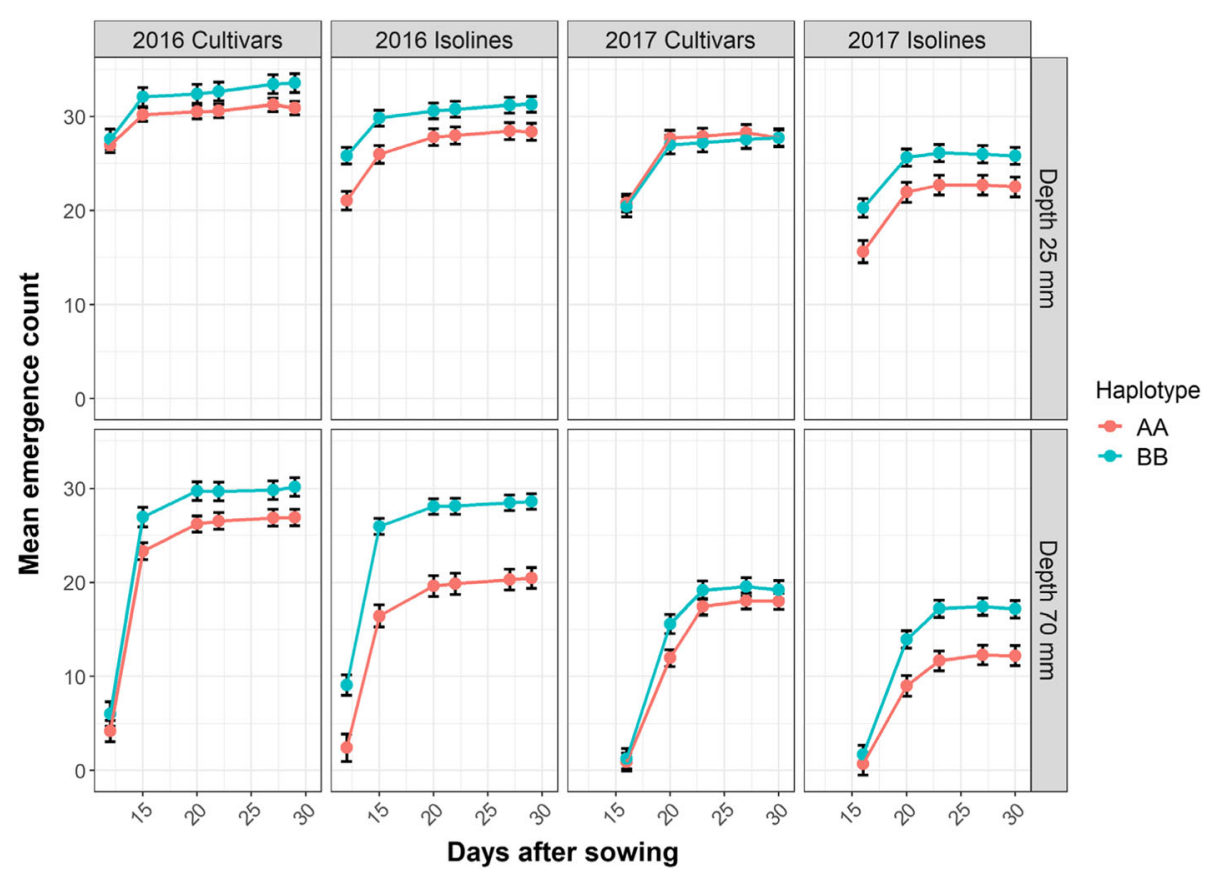

Fig. 6 Emergence (plants per linear meter) of isolines and cultivars with long (BB) and short (AA) Lcol-A1 alleles under normal (25 mm) and deep (70 mm) sowing in the field at Yanco in 2016 and 2017. Data are haplotype by depth by genotype class (Isolines vs Cultivars) means (obtained by averaging lines within a genotype class) \pm standard error

screened with markers IWB58229 and IWA710. Across all germplasm, the long Lcol-A1 containing haplotype was predicted to be present in $24 \%$, absent in $64 \%$, and recombinant in $12 \%$ (Additional file 2 : Table S2). An assessment of its evolution through the panel suggested that, despite the limited number of genotypes sampled in the early part of the twentieth century, Lcol-1A was reasonably common until 1960, but declined from 1960 to the latest cultivars released in 2015. To this end, the long Lcol-A1 allele was only present in $10 \%$ of the 56 varieties released from 2000 to 2015 (Fig. 7).

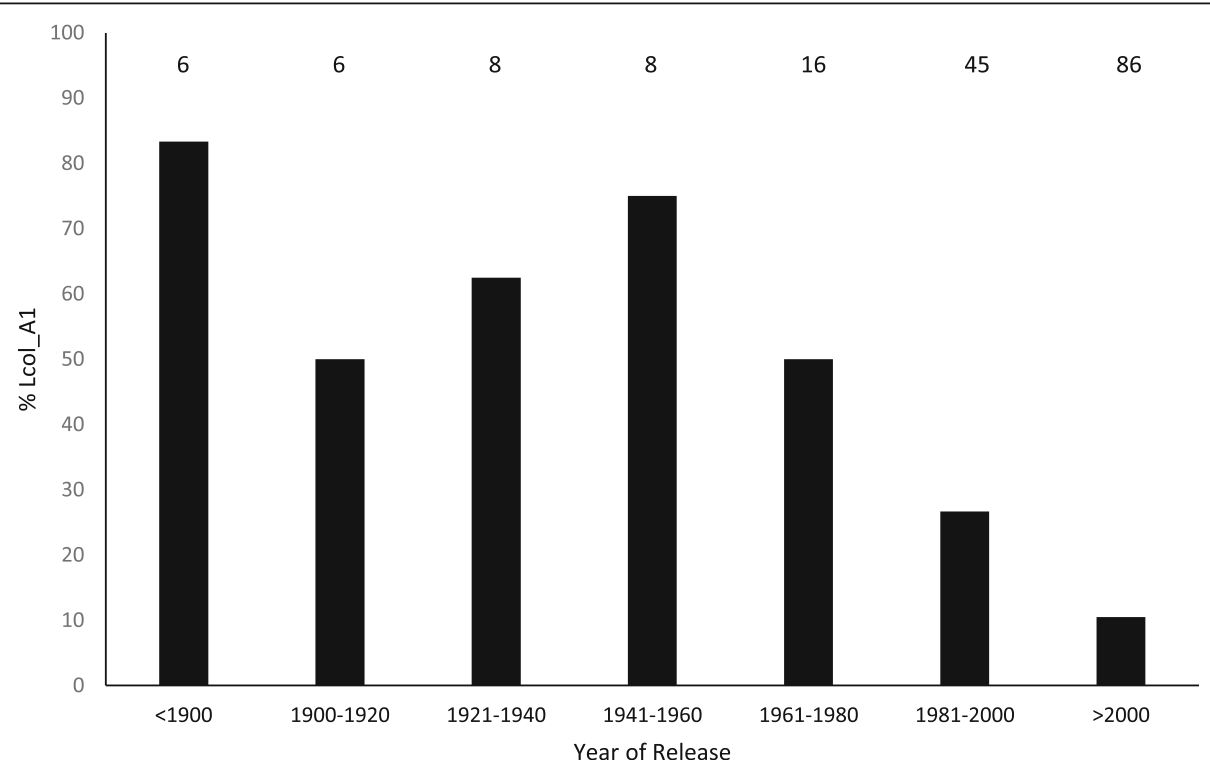

Fig. 7 Frequency of the long Lcol-Al allele in an Australian diversity panel. The number of cultivars genotyped from each time period is indicated above the bars 


\section{Discussion}

Good crop establishment presents challenges in many wheat growing regions of the world, and long coleoptile wheats are predicted to help mitigate constraints on emergence imposed by adoption of new management practices and a changing climate. This study characterised Lcol-A1, a gene which promotes coleoptile length and which is present in current Australian elite wheat germplasm. The gene is responsible for coleoptile length increases even in the presence of common DELLA dwarfing genes which translated to improved emergence with deep sowing in the field. Tightly linked SNP-based markers have been developed that can be used by breeders to assist in the selection process. Information about the gene and its effect under field conditions together with validated markers delivers a useful package to breeders and the industry and represents a step change from the background-specific marker-trait associations of small effect that were often generated in QTL studies.

A Young*3/H125M population ( $\mathrm{H} 125 \mathrm{M}$ is a Halberd derivative) was chosen for fine-mapping due to the large effect of Lcol-A1 and the clear phenotypic difference between homozygous classes. By coupling this genetic material to the fast and simple controlled environment screen used herein, we were able to delineate $L$ col-A1 to a $2 \mathrm{cM}$ region, which corresponded to a physical distance of approximately $7 \mathrm{Mb}$ in the RefSeqv1.0 genome sequence assembly [3]. Work is on-going to identify a candidate gene, prove its function and generate a gene-based marker for accurate selection in breeding programs. In the meantime, the flanking markers reported here will provide an efficient selection tool, particularly when flanking markers are combined to assay the Halberd-derived haplotype.

We showed that Lcol-A1 was effective in an Espada background, conferring an approximately $25 \mathrm{~mm}$ increase in coleoptile length. This validation was important, as it indicated that Lcol-A1 was effective in other genetic backgrounds additional to those used in the BSA and in the fine-mapping. Further evidence of the robustness of Lcol-A1 was provided from previous QTL mapping studies. Rebetzke et al. [33] identified a Halberdderived QTL for coleoptile length on 1AS, in a Cranbrook/Halberd Doubled Haploid population. In a 4-way MAGIC population, the largest effect QTL outside of the common DELLA dwarfing genes Rht-B1 and Rht-D1 was located on chromosome 1AS, inherited from cultivar Yitpi [35]. Yitpi and Halberd are related through pedigree, indicating that Yitpi carries $L c o l-A 1$ by descent. Confirmation of the effect of $L c o l-A 1$ in these populations provides further evidence of the utility of the gene across different genetic backgrounds.

Under conditions of deep sowing, Lcol-A1 was associated with an increase in emergence of up to approximately $40 \%$ in tall isolines, and of approximately $15 \%$ of cultivars carrying $R h t-B 1 b$ or $R h t-D 1 b$, confirming that the increase in coleoptile length afforded by Lcol-A1 translates to improved emergence. The consistent effect of Lcol-A1 detected in both the controlled environment screen with the field experiments confirms numerous reports about the positive relationship between coleoptile length and field emergence (e.g. [2, 42, 44]). The increased emergence under standard sowing depth with genotypes carrying Lcol-A1 indicates that the gene may also contribute to a faster rate of coleoptile growth. Other studies also showed that increased coleoptile length is associated with increased rates of emergence $[2,38]$. In Western Australia, a delay in sowing reduces yield by $28-36 \mathrm{~kg} \mathrm{ha}^{-1}$ day $^{-1}$ across germplasm with diverse sowing dates and phenology [39]; the increased emergence associated with Lcol-A1 may translate to reducing these yield losses by reducing the number of later emerging seedlings. Indeed, simulation studies by Kirkegaard and Hunt [25] and Flohr et al. [19] suggest that, when combined with appropriate management strategies, the long coleoptile trait can contribute substantially to farm yield.

Lcol-A1 is present in $\sim 20 \%$ of lines that were included in the Australian wheat diversity panel. When assessing the pedigree of Halberd (long coleoptile donor used in this study), it is possible that Lcol-A1 can be traced back to cultivars Steinwedel (a selection from the 1890's) and Federation (released in Australia in 1901; Additional file 3: Figure S1). Although Lcol-A1 was reasonably prevalent in Australian wheats until the 1960's, its presence appears to have decreased to only $10 \%$ of tested cultivars released since 2000. During the 1960's and 1970's DELLA dwarfing genes were introduced into Australia from CIMMYT, and CIMMYT derived material became dominant parents in Australian breeding programs [8]. None of the Mexican wheats from this era which were assayed with markers carried Lcol-A1, and so it is possible that the decline in $L c o l-A 1$ prevalence is the result of breeders preferring to use parental lines from CIMMYT over the past 50-60 years [24]. Alternatively, it may be that $L$ col-A1 is linked to a trait that Australian wheat breeders are unwittingly selecting against.

Bernando [6] laments the lack of impact for breeding from the many linkage mapping studies conducted to date, but results from mapping Lcol-A1 suggests otherwise for this trait. As opposed to novel loci from wild relatives and the challenge of reducing linkage drag when introgressing such loci, the Lcol-A1 haplotype is present in currently available elite varieties, which should aid in its transfer within breeding programs. Current work is underway to further refine the map location and to isolate the Lcol-A1 gene which will generate a gene-based marker. In the meantime, tightly linked and flanking SNP markers IWB58299 and IWA710 will 
be useful to predict the presence of the long allele of Lcol-A1 in diverse germplasm.

\section{Conclusions}

Establishment is a key phase in the life cycle of crops, and the length of the coleoptile significantly impacts upon establishment in wheat. As a result of changes in farming practices to earlier sowing into warmer soils, growers require that wheats with longer coleoptiles that are adapted to these changes are developed. Here, we confirm that Lcol-A1 increases coleoptile length which results in improved emergence under field conditions. By developing tightly linked flanking markers, we have provided tools to allow the wheat breeding community to incorporate $\mathrm{Lcol}$ A1 more efficiently in future cultivars.

\section{Methods}

\section{Phenotyping for coleoptile length - controlled environment}

For coleoptile length measurements, 14 seeds/line were sown in $100 \mathrm{~mm}$ deep wooden trays (50:50 fertile compost/vermiculite soil mix) and grown in growth cabinets at $15^{\circ} \mathrm{C}$ for 14 days (210 degree days) in the dark before coleoptile lengths were measured with a ruler.

\section{Bulked-segregant analysis}

One-hundred and eight-eight (188) $\mathrm{BC}_{1} \mathrm{~F}_{2}$ progeny from a Halberd*2/Chuan-Mai18 (CM18) population (Halberd is an older Australian wheat with a long coleoptile, while CM18 is a Chinese wheat with a short coleoptile [41] and $188 \mathrm{BC}_{1} \mathrm{~F}_{2}$ progeny from a Uruguay386*2/CM18 population (a tall Uruguay accession (AUS1517) from the Australian Winter Cereals Collection with a long coleoptile) were phenotyped for coleoptile length to identify 10 short and 10 long coleoptile lines from each population. DNA from each class was pooled separately for each population and genotyped by BSA using the $9 \mathrm{~K}$ SNP array at Agriculture Victoria Research, La Trobe University [10]. These populations were used for BSA only and not progressed for mapping.

\section{Genetic mapping of the long coleoptile gene}

Two backcross populations developed from HI25M (long coleoptile Halberd derivative) and Young (short coleoptile cultivar) were used to map the long coleoptile gene. Firstly, 434 $\mathrm{BC}_{1} \mathrm{~F}_{2}$ plants were screened with a marker derived from the Rht-B1b dwarfing allele [13] to identify $54 \mathrm{BC}_{1} \mathrm{~F}_{2}$ plants that lacked Rht-B1b. The elimination of the known dwarfing gene at an early stage resulted in tall progeny with only minor height variation that was derived from this population for fine mapping. These plants were genotyped with marker IWA164 which was mapped in the $9 \mathrm{~K}$ SNP consensus map of wheat [10] to the 1A region identified in $\mathrm{BSA}$, and $\mathrm{BC}_{1} \mathrm{~F}_{3}$ progeny were phenotyped for coleoptile length under controlled conditions (see above). Similarly, the plants were genotyped with markers IWA3374 and IWA5435 from the $9 \mathrm{~K}$ SNP consensus map, which flanked the $1 \mathrm{~A}$ region identified in $\mathrm{BSA}$. The $\mathrm{BC}_{1}$ derived population was used to map the long coleoptile gene to chromosome $1 \mathrm{~A}$ and to identify flanking SNP-based markers that were critical for the next mapping step.

To increase the resolution of the target region, $\mathrm{BC}_{2} \mathrm{~F}_{3}$ progeny from $\mathrm{BC}_{2} \mathrm{~F}_{2}$ plants that were heterozygous for markers in the $1 \mathrm{~A}$ region were screened with flanking markers IWA3374 and IWA5435. DNA was extracted from half-seeds of $900 \mathrm{BC}_{2} \mathrm{~F}_{3}$ individuals using the protocol of Ellis et al. [14]. To make the screening process more efficient, SNP-based markers were converted to Kompetitive allele specific PCR (KASP) assays [22].

Plants from half seed that carried recombination events between the flanking markers were selfed. Twelve to 16 progeny were genotyped to identify individual plants that were recombinant and homozygous in the target region. Coleoptile length was measured in homozygous progeny which were used to fine map the gene with additional $90 \mathrm{~K}$ SNP markers that were polymorphic between Halberd and Young, developed from the consensus map of the region.

\section{Validation of the $1 \mathrm{~A}$ region}

The 1A region was transferred from HI25M to Espada (an Australian cultivar) through backcrossing. $\mathrm{BC}_{3} \mathrm{~F}_{3}$ plants were genotyped for Rht-D1b [13], Rht18 [21], and the 1A locus. Lines that were homozygous wild-type for Rht-D1, homozygous dwarf for Rht18, and heterozygous for the $1 \mathrm{~A}$ locus were selfed to produce $\mathrm{BC}_{3} \mathrm{~F}_{3}$ derived near isogenic lines (NILs). Coleoptile length of the NILs was phenotyped using the method described above, and the same plants genotyped to assess marker-trait associations.

\section{Field performance}

To validate the $1 \mathrm{~A}$ locus across different genetic backgrounds and to provide evidence that the increase in coleoptile length improves emergence under field conditions, two field experiments were conducted at Yanco (34 $36^{\prime}$ 53.6"S 146 24' 54.2"E) in Southern New South Wales, Australia in 2016 and 2017. A split-plot randomised block design balanced for row and column and consisting of four replicates of 10 tall isolines (five short and five long coleoptile haplotype) of $\mathrm{BC}_{1} \mathrm{~F}_{5}$ plants from the Young*3/H125M population as well as 11 diverse Australian cultivars (six short and five long coleoptile 1A haplotype) were sown at depths of $25 \mathrm{~mm}$ and $70 \mathrm{~mm}$ (main plots) in plots that were $7 \mathrm{~m}$ long and 8 rows wide, with $20 \mathrm{~cm}$ row spacing. Emerged plants (plants per linear meter) were counted in four rows per plot 12, 15, 20, 22, 27, and 29 days after sowing in 2016, and 16, 20,23,27, and 30 days after sowing in 2017. Sowing depth in the deep sowing treatment was 
checked by measuring the coleoptile length in seedlings after they emerged. Cultivars carried either Rht-B1b or Rht$D 1 b$ dwarfing gene and were similar in final plant height. They were chosen based on their adaptation to different climatic zones of the Australian wheat belt.

\section{Allele survey and pedigree assessment}

To assess the frequency of the 1A haplotype from Halberd across diverse germplasm, DNA from an Australian diversity panel comprised of 197 bread wheat cultivars and lines was assayed with markers IWB58229 and IWA710. To assess pedigree relationships of lines sharing a common haplotype, the Genetic Resources Information System for Wheat and Triticale (GRIS; wheatpedigree.net) was queried.

\section{Statistical analyses}

The controlled environment and the 2016 and 2017 field experiment datasets were analysed using the linear mixed model software asreml [9] for $R$ ( $R$ [29]).

For the controlled environment experiment, cultivar and isoline genotype class groups were analysed separately. To compare haplotypes, the traditional 'one-way ANOVA' analysis for comparing two treatment groups was extended to a linear mixed model by representing varieties comprising the particular genotype class as a random effects factor in the statistical model, thus ensuring the correct degrees of freedom were used for the haplotype comparison. F-tests for the significance of the haplotype term yield the $p$-values for the comparison between AA and BB haplotypes within each genotype class. Potential outliers were identified using the $1.5 \mathrm{x}$ interquartile range criterion [4].

The field experiments in 2016 and 2017 were analysed separately, although isolines and cultivars were analysed together within each trial. The field experiments involve factorial treatment structures for haplotype, genotype class and sowing depth, modelled as fixed effects. Since the haplotype/genotype class combinations represent groups of varieties, variety was included in the model as a nested random effects factor, and variety interaction terms were therefore modelled as random effects terms. Further random effects terms were included to represent the spatial structure of the trial layout, namely block, plot and subplot (representing row within plot). The models incorporated the recording of observations across multiple dates by accounting for autocorrelation structure across the (unevenly spaced) dates, and allowing heterogeneity of the residual variances across dates. For the 2016 dataset the model also allowed for unequal variances that were observed between the two sowing depths.

\section{Additional files}

Additional file 1: Table S1. All significant marker-trait associations detected from BSA using the $9 \mathrm{~K}$ SNP array in the Halberd*2/CM18 and Uruguay*2/CM18 populations. (DOCX $29 \mathrm{~kb}$ )

Additional file 2: Table S2. Genotyping results from the Australian diversity panel. Cultivar, year of release, and allele call (A indicates short allele; B indicates long allele) for markers IWB58229 and IWA710 are shown. (DOCX $31 \mathrm{~kb}$ )

Additional file 3: Figure S1. Pedigree of Halberd. Cultivars which were genotyped in the diversity panel carrying the long (green) or short (yellow) Lcol-A1 alleles are highlighted. Gaza, a cultivar carrying a recombinant haplotype is highlighted in orange. (DOCX $270 \mathrm{~kb}$ )

\section{Abbreviations}

AFLP: Amplified fragment length polymorphism; BSA: Bulked-segregant analysis; GWAS: Genome wide association study; KASP: Kompetitive allele specific PCR; MAGIC: Multi-parent advanced generation intercross; MTA: Marker trait association; QTL: Quantitative trait locus; SNP: Single nucleotide polymorphism

\section{Acknowledgements}

We are grateful to Kathryn Bechaz, Dionne Wornes and Peter Davidson for technical assistance for the field experiments at Yanco and to Drs. Dianne Mather, Shannon Dillon and Ben Trevaskis for providing DNA used to construct the diversity panel.

\section{Authors' contributions}

Wrote the manuscript, developed germplasm and performed experiments: WDB. Performed experiments and reviewed the manuscript: JH. Performed statistical analyses and reviewed the manuscript: ABZ. Performed finemapping experiments and reviewed the manuscript: BAF. Developed germplasm and reviewed the manuscript: BJB and GJR. Performed experiments: GP and TP. Undertook $9 \mathrm{~K}$ SNP BSA analysis and reviewed the manuscript: $\mathrm{MJH}$. Oversaw field experiments and reviewed the manuscript: JRH. Conceived, supervised the experiments, contributed to manuscript writing and revision: WS. All authors read and approved the final manuscript.

\section{Funding}

This research was supported financially by the Grains Research and Development Corporation (GRDC project CSP00200).

\section{Availability of data and materials}

The datasets used and/or analysed during the current study are available from the corresponding author on reasonable request.

Ethics approval and consent to participate Not applicable.

\section{Consent for publication}

Not applicable.

\section{Competing interests}

The authors declare that they have no competing interests.

\section{Author details}

${ }^{1}$ CSIRO Agriculture and Food, P.O. Box 1700, Canberra ACT 2601, Australia. 2Data61, CSIRO, P.O. Box 1700, Canberra ACT 2601, Australia. ${ }^{3}$ Agriculture Victoria Research, AgriBio Centre for AgriBiosciences, Bundoora, VIC 3086, Australia. ${ }^{4}$ Department of Animal, Plant and Soil Sciences, AgriBio Centre for AgriBiosciences, La Trobe University, Bundoora, VIC 3086, Australia.

Received: 4 March 2019 Accepted: 2 July 2019

Published online: 29 July 2019

\section{References}

1. Allan RE. Influence of semidwarfism and genetic background on stand establishment of wheat. Crop Sci. 1980;20:634-8. 
2. Allan RE, Vogel OA, Peterson CJ. Seedling emergence rate of fall-sown wheat and its association with plant height and coleoptile length. Agron J. 1962;54:347-50

3. Appels R, The International Wheat Genome Sequencing Initiative (IWGSC). Shifting the limits in wheat research and breeding using a fully annotated reference genome. Science. 2018;345:1251788.

4. Barbato G, Barini EM, Genta G, Levi R. Features and performance of some outlier detection methods. J Appl Stat. 2011;38:2133-49.

5. Beharav A, Cahaner A, Pinthus MJ. Genetic correlations between culm length, grain yield and seedling elongation within tall (rht1) and semi-dwarf (Rht1) spring wheat (Triticum aestivum L.). Eur J Agron. 1998:9:35-40.

6. Bernardo R. Bandwagons I, too, have known. Theor Appl Genet. 2016;129:2323-32

7. Bhatt GM, Sheedi SM. Sensitivity of wheat coleoptile to variation in temperature. Cereal Res Commun. 1986;14:155-60.

8. Brennan JP, Quade KJ. Analysis of the impact of CIMMYT research on the Australian wheat industry. Wagga Wagga: Economic research report no. 25, NSW Department of Primary Industries, Wagga Wagga; 2004.

9. Butler DG. 2009. Asreml: asreml() fits the linear mixed model. $R$ package version 3.0. http://www.vsni.co.uk.

10. Cavanagh CR, Chao S, Wang S, Huang BE, Stephen S, Kiani S, Forrest K, Saintenac C, Brown-Guedira GL, Akhunova A, See D, Bai G, Pumphrey M, Tomar L, Wong D, Kong S, Reynolds M, da Silva ML, Bockelman H, Talbert L, Anderson JA, Dreisigacker S, Baenziger S, Carter A, Korzun V, Morrell PL, Dubcovsky J, Morell MK, Sorrells ME, Hayden MJ, Akhunov E. Genome-wide comparative diversity uncovers multiple targets of selection for improvement in hexaploid wheat landraces and cultivars. Proc Natl Acad Sci. 2013:110:8057-62.

11. Collis-George N, Yoganathan P. The effect of soil strength on germination and emergence of wheat (Triticum aestivum L.). I. Low shear strength conditions. Soil Research. 1985a;23:577-87.

12. Collis-George N, Yoganathan P. The effect of soil strength on germination and emergence of wheat (Triticum aestivum L.). II. High shear strength conditions. Soil Research. 1985b;23:589-601.

13. Ellis M, Spielmeyer W, Gale K, Rebetzke G, Richards R. "perfect" markers for the Rht-B1b and Rht-D1b dwarfing genes in wheat. Theor Appl Genet. 2002;105:1038-42.

14. Ellis MH, Rebetzke GJ, Azanza F, Richards RA, Spielmeyer W. Molecular mapping of gibberellin-responsive dwarfing genes in bread wheat. Theor Appl Genet. 2005;111:423-30.

15. Feather J, Qualset C, Vogt H. Planting depth critical for short-statured wheat varieties. Calif Agric. 1968;22:12-4

16. Fletcher A, Lawes R, Weeks C. Crop area increases drive earlier and dry sowing in Western Australia: implications for farming systems. Crop Pasture Sci. 2016:67:1268-80.

17. Fletcher AL, Robertson MJ, Abrecht DG, Sharma DL, Holzworth DP. Dry sowing increases farm level wheat yields but not production risks in a Mediterranean environment. Agric Syst. 2015;136:114-24.

18. Flohr BM, Hunt JR, Kirkegaard JA, Evans JR. Water and temperature stress define the optimal flowering period for wheat in South-Eastern Australia. Field Crop Res. 2017;209:108-19.

19. Flohr BM, Hunt JR, Kirkegaard JA, Evans JR, Lilley JM. Genotype $\times$ management strategies to stabilise the flowering time of wheat in the south-eastern Australian wheatbelt. Crop Pasture Sci. 2018b;69:547-60.

20. Flohr BM, Hunt JR, Kirkegaard JA, Evans JR, Trevaskis B, Zwart A, Swan A, Fletcher AL, Rheinheimer B. Fast winter wheat phenology can stabilise flowering date and maximise grain yield in semi-arid Mediterranean and temperate environments. Field Crop Res. 2018a;223:12-25.

21. Ford BA, Foo E, Sharwood R, Karafiatova M, Vrána J, MacMillan C, Nichols DS, Steuernagel B, Uauy C, Doležel J, Chandler PM, Spielmeyer W. Rht18 semidwarfism in wheat is due to increased GA 2-oxidaseA9 expression and reduced GA content. Plant Physiol. 2018;177:168-80.

22. He C, Holme J, Anthony J. SNP genotyping: the KASP assay. In: Fleury D, Whitford R, editors. Crop breeding: methods and protocols. Springer New York: New York, NY; 2014. p. 75-86.

23. Hedden P. The genes of the green revolution. Trends Genet. 2003;19:5-9.

24. Joukhadar R, Daetwyler HD, Bansal UK, Gendall AR, Hayden MJ. Genetic diversity, population structure and ancestral origin of Australian wheat. Front Plant Sci. 2017:8:2115.

25. Kirkegaard JA, Hunt JR. Increasing productivity by matching farming system management and genotype in water-limited environments. J Exp Bot. 2010;61:4129-43.
26. Li G, Bai G, Carver BF, Elliott NC, Bennett RS, Wu Y, Hunger R, Bonman JM, Xu X. Genome-wide association study reveals genetic architecture of coleoptile length in wheat. Theor Appl Genet. 2017;130:391-401.

27. Li P, Chen J, Wu P, Zhang J, Chu C, See D, Brown-Guedira G, Zemetra R, Souza E. Quantitative trait loci analysis for the effect of Rht-B1 dwarfing gene on coleoptile length and seedling root length and number of bread wheat. Crop Sci. 2011;51:2561-8.

28. Lilley JM, Kirkegaard JA. Farming system context drives the value of deep wheat roots in semi-arid environments. J Exp Bot. 2016;67:3665-81.

29. Core Team R. R: a language and environment for statistical computing. Vienna: R Foundation for Statistical Computing; 2017. https://www.r-project.org/

30. Radford B. Effect of constant and fluctuating temperature regimes and seed source on the coleoptile length of tall and semidwarf wheats. Aust J Exp Agric. 1987;27:113-7

31. Rebetzke GJ, Appels R, Morrison AD, Richards RA, McDonald G, Ellis MH, Spielmeyer W, Bonnett DG. Quantitative trait loci on chromosome 4B for coleoptile length and early vigour in wheat (Triticum aestivum L.). Aust J Agric Res. 2001;52:1221-34

32. Rebetzke GJ, Bruce SE, Kirkegaard JA. Longer coleoptiles improve emergence through crop residues to increase seedling number and biomass in wheat (Triticum aestivum L.). Plant Soil. 2005;272:87-100.

33. Rebetzke GJ, Ellis MH, Bonnett DG, Richards RA. Molecular mapping of genes for coleoptile growth in bread wheat (Triticum aestivum L.). Theor Appl Genet. 2007;114:1173-83.

34. Rebetzke GJ, Richards RA, Sirault XRR, Morrison AD. Genetic analysis of coleoptile length and diameter in wheat. Aust J Agric Res. 2004;55:733-43.

35. Rebetzke GJ, Verbyla AP, Verbyla KL, Morell MK, Cavanagh CR. Use of a large multiparent wheat mapping population in genomic dissection of coleoptile and seedling growth. Plant Biotechnol J. 2014;12:219-30.

36. Rebetzke GJ, Zheng B, Chapman SC. Do wheat breeders have suitable genetic variation to overcome short coleoptiles and poor establishment in the warmer soils of future climates? Funct Plant Biol. 2016;43:961-72.

37. Sayre KD, Rajaram S, Fischer RA. Yield potential progress in short bread wheats in Northwest Mexico. Crop Sci. 1997;37:36-42.

38. Schillinger WF, Donaldson E, Allan RE, Jones SS. Winter wheat seedling emergence from deep sowing depths. Agron J. 1998;90:582-6.

39. Shackley $B$, Anderson W. Responses of wheat cultivars to time of sowing in the southern wheatbelt of Western Australia. Aust J Exp Agric. 1995;35:579-87.

40. Singh K, Shukla S, Kadam S, Semwal VK, Singh NK, Khanna-Chopra R. Genomic regions and underlying candidate genes associated with coleoptile length under deep sowing conditions in a wheat RIL population. J Plant Biochem Biotechnol. 2015;24:324-30.

41. Spielmeyer W, Hyles J, Joaquim P, Azanza F, Bonnett D, Ellis ME, Moore C, Richards RA. A QTL on chromosome 6A in bread wheat (Triticum aestivum) is associated with longer coleoptiles, greater seedling vigour and final plant height. Theor Appl Genet. 2007;115:59-66.

42. Sunderman DW. Seedling emergence of winter wheats and its association with depth of sowing, coleoptile length under various conditions, and plant height. Agron J. 1964;56:23-5.

43. Wang S, Wong D, Forrest K, Allen A, Chao S, Huang BE, Maccaferri M, Salvi S, Milner SG, Cattivelli L, Mastrangelo AM, Whan A, Stephen S, Barker G, Wieseke R, Plieske J, International Wheat Genome Sequencing C, Lillemo M, Mather D, Appels R, Dolferus R, Brown-Guedira G, Korol A, Akhunova AR, Feuillet C, Salse J, Morgante M, Pozniak C, Luo M-C, Dvorak J, Morell M, Dubcovsky J, Ganal M, Tuberosa R, Lawley C, Mikoulitch I, Cavanagh C, Edwards KJ, Hayden M, Akhunov E. Characterization of polyploid wheat genomic diversity using a high-density 90000 single nucleotide polymorphism array. Plant Biotechnol J. 2014;12:787-96.

44. Whan B. The emergence of semidwarf and standard wheats, and its association with coleoptile length. Aust J Exp Agric. 1976;16:411-6.

45. Yu J-B, Bai G-H. Mapping quantitative trait loci for long coleoptile in Chinese wheat landrace Wangshuibai. Crop Sci. 2010;50:43-50.

\section{Publisher's Note}

Springer Nature remains neutral with regard to jurisdictional claims in published maps and institutional affiliations. 